\title{
Trafficking Inhibition of Bruceanol B as a Radical-Producing Antibiotic
}

Doseung Lee $\cdot$ Kyung-Hwan Boo $\cdot$ Jin-Kyu Woo $\cdot$ Quanchun Hong

Somi K. Cho $\cdot$ Se Pill Park $\cdot$ Dong-Sun Lee $\cdot$ Key Zung Riu

\begin{abstract}
Acknowledgments. This work was supported by the Basic Science Research Program through the National Research Foundation of Korea (NRF) (2010-0477), the Gene and Material Bank for Citrus Breeding at Jeju National University (KRF-2007-412-J05502), Priority Research Centers Program (2010-0029630), and Mid-career Researcher Program (2010-0027752) through the National Research Foundation of Korea (NRF) funded by the Ministry of Education, Science and Technology.
\end{abstract}

The above article was corrected with concision as follows:

Acknowledgments. This work was supported by the Basic Science Research Program through the National Research Foundation of Korea (NRF) (2010-0012641), the Gene and Material Bank for Citrus Breeding at Jeju National University (KRF-2007-412-J05502), Priority Research Centers Program (2010-0029630), and Mid-career Researcher Program (2010-0027752) through the National Research Foundation of Korea (NRF) funded by the Ministry of Education, Science and Technology. 